\title{
End-of-life home care during the COVID-19 pandemic
}

Ryo Sakamoto ${ }^{1}$, Divya Bhandari ${ }^{2}$, Akihiko Ozaki ${ }^{3}$, Hirotomo Miyatake ${ }^{4}$, Makoto Kosaka ${ }^{2}$, Makoto Yoshida ${ }^{5}$, Tetsuya Tanimoto ${ }^{2}$, and Masahiro Kami ${ }^{2}$

${ }^{1}$ Visina Home care

${ }^{2}$ Medical Governance Research Institute

${ }^{3}$ Joban Byoin

${ }^{4}$ Orange Home-Care Clinic

${ }^{5}$ Teikyo Daigaku

October 21, 2021

\begin{abstract}
Patients and their families are discouraged from the restrictions on hospital visits for coronavirus Infection control. This separation might further deteriorate the mental health of both vulnerable patients and their family members. Home care could be a preferable solution to this problem.
\end{abstract}

Title: End-of-life home care during the COVID-19 pandemic

\section{Co-authors:}

1. Ryo Sakamoto

Departments:

Institutions: Visina Home Care

City: Tokyo

Country: Japan

Email:ryo.sakamoto1227@gmail.com

2. Divya Bhandari

Departments:

Institutions: Medical Governance Research Institute

City: Tokyo

Country: Japan

Email: rayordeal3@gmail.com

3. Akihiko Ozaki

Departments: Department of Breast Surgery

Institutions: Jyoban Hospital of Tokiwa Foundation

City: Fukushima 
Country: Japan

Email:ozakiakihiko@gmail.com

4. Hirotomo Miyatake

Departments:

Institutions: Orange Home-Care Clinic

City: Fukui

Country: Japan

Email:hmiyatake@orangeclinic.jp

5. Makoto Kosaka

Departments:

Institutions: Medical Governance Research Institute

City: Tokyo

Country: Japan

Email:m.kosaka0811@gmail.com

6. Makoto Yoshida

Departments:

Institutions: Medical Governance Research Institute

City: Tokyo

Country: Japan

Email:110818makoto@gmail.com

7. Tetsuya Tanimoto

Departments:

Institutions: Medical Governance Research Institute

City: Tokyo

Country: Japan

Email: tetanimot@yahoo.co.jp

8. Masahiro Kami

Departments:

Institutions: Medical Governance Research Institute

City: Tokyo

Country: Japan

Email:kami-tky@umin.net

Corresponding author:

Ryo Sakamoto 
Postal address: Minatoku, Tokyo, Japan

Email: ryo.sakamoto1227@gmail.com

Tel: +819082761399

Fax: 03-6409-6358

\section{DATA AVAILABILITY STATEMENT}

Data sharing is not applicable to this article as no datasets

were generated or analyzed during current study.

\section{Funding Statement:}

None.

\section{Conflict of interest disclosure:}

AO reports personal fees from Medical Network Systems, MNES Inc. TT reports

personal fees from Medical Network Systems, MNES Inc. and Bionics co. Itd. Other

authors declare no competing interests.

Ethics approval statement:

Written informed consent was obtained from the patient's child.

Patient consent statement:

The patient is deceased and we obtained a consent form from the patient's child.

Permission to reproduce material from other sources:

Not applicable.

\section{Clinical trial registration:}

Not applicable.

\section{Abstract:}

Patients and their families are discouraged from the restrictions on hospital visits for coronavirus Infection control. This separation might further deteriorate the mental health of both vulnerable patients and their family members. Home care could be a preferable solution to this problem.

\section{Clinical message:}

The findings of this case study highlight that in crises situation such as COVID-19, home care could be considered as a viable option for terminally ill cancer patients.

\section{Introduction}

End-of-life care for cancer patients is imperative as it aims to optimize the quality of life by alleviating physical, social, spiritual, and psychological suffering experienced throughout the dying process. Proper interaction/communication between terminally ill patients and their family members is particularly crucial because it enables a patient to adjust to the process with ease and family members to move on after death with mitigated regret (1). Such provisions have been made available and are promoted in hospices and palliative care wards.

However, the current coronavirus disease 2019 (COVID-19) pandemic has affected various aspects of healthcare, and end-of-life care is no exception. In an attempt to prevent the spread of COVID-19 infection, patients' families are discouraged from visiting hospitals and nursing homes. This separation might further 
deteriorate the mental health of both vulnerable patients and their family members (2). That being said, providing effective end-of-life care in medical institutions amid the pandemic to cancer patients who are highly susceptible to the infection itself has been a major challenge worldwide.

Alternatively, home care could be a preferable solution to this problem. Home care is a form of medical care in which patients stay at home with the help of their families and medical professionals. This form of care is actively practiced in some countries, including Japan, where reimbursement for home care is available, including for cancer patients. In Japan, the first infection of COVID-19 was noted in January 2020, and a state of emergency was subsequently declared from April 2020 to May 2020. Since then, the infection has not been well controlled yet. Until now, many medical institutions have implemented restrictions on hospitalized patients.

In this article, we present a case study of a Japanese patient who spent the final stages of his illness at home using home care during the COVID-19 pandemic.

\section{Case presentation}

A man in his late 80s living in Tokyo with his wife and two daughters experienced sudden abdominal pain in July 2020 and went to seek help in a nearby tertiary hospital. That same day, he had to undergo an emergency laparotomy and appendicectomy after being diagnosed with generalized peritonitis associated with perforation of appendiceal cancer. Once the diagnostic tests were conducted, the patient was confirmed to have Stage IV appendiceal cancer. Postoperative contrast-enhanced CT scan showed no abnormal findings such as local recurrence, lymphadenopathy, or distant metastasis associated with cancer, but histopathological tests showed scattered lymphatic invasion in the sub-serous layer.

Family members were informed about the severity of the disease highlighting the need for palliative care. However, due to restrictions imposed by the hospital amid COVID-19, the family members were not allowed to visit frequently with the patients. Accordingly, they started seeking updates on the patient's condition through phone calls with the medical staff. However, as family members were unable to understand all situations/information clearly, there were few instances of misunderstanding and even conflict between medical staffs and family members. For example, there was an incident where the family members thought that the intravenous drip was administered through the peripheral intravenous catheter, but later they found it was administered through the peripherally inserted central venous catheter. This kind of incident was troublesome for family members, and they were particularly concerned when medical staff asked them not to call too often and told that medical staff would contact them directly if anything happens to the patients.

Considering the patient's desire to spend his final period at home with his family and the restrictions on hospital visits due to pandemic, the patient and his family chose to receive home care in November 2020. After his discharge, home-care nurses visited twice a day and the home doctor visited once every two weeks. This allowed the family to spend enough time with the patient, which was not possible in the hospital due to the restriction. Two daughters assisted his wife as family caregivers, and professional caregivers provided physical care through public nursing care insurance scheme. On weekends, his grandchildren and greatgrandchildren also visited him, and the whole family was able to spend quality time with the terminally ill patient. Moreover, communication between the medical staff and the family was also enhanced as nurses used to communicate on a daily basis, and the doctor in charge used to make house calls as needed. Finally, in April 2021, the patient passed away peacefully after experiencing acute respiratory distress syndrome (ARDS) caused by heart failure.

\section{Discussion}

This case illustrates that home care can be a viable option in end-of-life care for cancer patients, particularly during crises such as the recent COVID-19 pandemic. Globally, a standard set of end-of-life care is a hospice or palliative care ward which aimed to provide holistic care to patients. While focusing on personalized care of patients, hospice also seeks to ameliorate the tolls and hardship faced by the family caregivers while taking care of their patients (3). In contrast, this case demonstrated that home care would allow terminally 
ill patients to stay where they feel most comfortable so that they could cherish their lifetime memories and spend quality time with their loved ones.

We believe that there are several other reasons to choose for home care for end-of-life care during a COVID-19 pandemic. First, this would alleviate communication problems between health care professionals and family members. Restriction imposed due to COVID-19 has been reported to reduce the quality of communication between hospitalized patients and their family members, resulting in severe distress that may affect the quality of death and bereavement $(4,5)$. Second, home care would potentially ease the burden relating to COVID-19 care among medical professionals in hospitals. End-of-life patients are considered a vulnerable population and infection with SARS-CoV2 can result in the development of serious illness. In addition to the risk of nosocomial infection, work overload of these staff and logistic shortage are also an issue. Given these reasons, home care could be considered as a viable option at least for a while until the pandemic subsides.

However, home care also has its drawbacks. Health workers are not easily available and it is more difficult to address patient's health problems in a timely manner compared to hospitalization. Further, there might be a risk of COVID-19 infection due to frequent visits from health workers, families, and relatives. In any case, patients must be informed about the advantages and disadvantages of both hospice and home care so that they can make their own choices autonomously.

\section{Conclusion}

The findings of this case study highlight that in crises situation such as COVID-19, home care could be considered as a viable option for terminally ill cancer patients.

\section{Author Contributions:}

All authors conceptualized and designed the study. Sakamoto R, Bhandari D, Ozaki A and Tanimoto T wrote the manuscript, and all authors contributed to making critical revisions for improving the intellectual content of the manuscript.

Acknowledgments:

None.

\section{References:}

1. Boyle DK, Miller PA, Forbes-Thompson SA. Communication and end-of-life care in the intensive care unit: patient, family, and clinician outcomes. Crit Care Nurs Q. 2005;28(4):302-16.

2. Rosenstein DL. Depression and end-of-life care for patients with cancer. Dialogues Clin Neurosci. 2011;13(1):101-8.

3. Kris AE, Cherlin EJ, Prigerson H, Carlson MD, Johnson-Hurzeler R, Kasl SV, et al. Length of hospice enrollment and subsequent depression in family caregivers: 13-month follow-up study. Am J Geriatr Psychiatry. 2006;14(3):264-9.

4. Feder S, Smith D, Griffin H, Shreve ST, Kinder D, Kutney-Lee A, et al. "Why Couldn't I Go in To See Him?" Bereaved Families' Perceptions of End-of-Life Communication During COVID-19. J Am Geriatr Soc. 2021;69(3):587-92.

5. Otani H, Yoshida S, Morita T, Aoyama M, Kizawa Y, Shima Y, et al. Meaningful Communication Before Death, but Not Present at the Time of Death Itself, Is Associated With Better Outcomes on Measures of Depression and Complicated Grief Among Bereaved Family Members of Cancer Patients. J Pain Symptom Manage. 2017;54(3):273-9. 